\title{
Radiological evaluation of post-tracheostomy lesions
}

\author{
ALEXANDER S. MACMILLAN, A. EVERETTE JAMES, JR., \\ FREDERICK P. STITIK, and HERMES C. GRILLO
}

Departments of Radiology, Massachusetts Eye and Ear Infirmary, Boston, Mass. and Johns Hopkins Hospital. Baltimore, Maryland and Division of Thoracic Surgery, Massachusetts General Hospital, Boston, Mass., U.S.A.

\begin{abstract}
Post-tracheostomy lesions are becoming more commonplace and surgical techniques for definitive repair of these abnormalities are being developed. These lesions, in general, occur at two sites, the proximal lesions at the tracheostomy incision and the distal lesions 1.5 to $2.5 \mathrm{~cm}$ inferior in the area of the tracheostomy balloon cuff. Granuloma formation, stenosis, tracheomalacia, and perforation of the tracheal wall have been encountered in our experience. Clinical symptoms depend upon the type and location of the lesion as well as on the patient's awareness and physical activity. Radiological evaluation offers an accurate method to depict the anatomical and physiological alterations. This radiological assessment should begin with routine postero-anterior and lateral chest radiographs followed by fluoroscopy. Laminograms and special oblique views are often helpful. Contrast tracheograms using powdered tantalum allow good mucosal detail as well as excellent delineation of structural and physiological abnormalities.
\end{abstract}

The more common use of various methods of assisted respiration coupled with the many modifications of tracheostomy tubes has led to an increased incidence of post-tracheostomy complications. Preoperative evaluation of these lesions should include delineation and documentation of anatomical alterations as well as assessment of the significance of the physiological abnormalities. Many of these determinations can be made by radiological methods. This communication addresses itself to the orderly sequence of radiological evaluation of these problems, emphasizing the expected risk and potential value of each type of examination.

\section{MATERIALS AND METHODS}

Because of the particular surgical interest of one of us (H.C.G.), a large number of patients were referred to the Massachusetts Eye and Ear Infirmary and Massachusetts General Hospital for evaluation and definitive repair of posttracheostomy lesions. We have also been interested in a new contrast agent for radiographic evaluation of post-tracheostomy lesions and one of us (F.P.S.) has recently engaged in research and clinical evaluation of the use of powdered tantalum. From this experience (1966-71) a flexible protocol for evaluation of post-tracheostomy lesions has evolved and is presented.

\section{POST-TRACHEOSTOMY LESIONS}

In the radiological evaluation of post-tracheostomy complications, the expected sequence and pathological alterations must be kept in mind. Proper radiological studies should be requested accordingly. Generally, the lesions will occur in two sites in the trachea-at the site of the tracheostomy incision and in the area of the tracheostomy balloon cuff. The proximal lesions occurring at the tracheostomy incision sites will most often involve the anterior and lateral tracheal walls and will consist of stenosis, granulation mass formation, and, occasionally, tracheomalacia (Meade, 1961; Murphy, MacLean, and $D$ Dobell. 1966). Those distal post-tracheostomy lesions which occur in the area of the balloon $N$ cuff consist of (Fig. 1) stenosis, granulation mass formation, tracheomalacia, and perforation $\tilde{N}$ (Pearson, Goldberg, and da Silva, 1968 ; Stiles, 1965).

The histological changes of both proximal and 0 distal tracheal lesions are comparable and may be $\stackrel{\overparen{D}}{\overparen{D}}$ concomitantly discussed. After placement of the $\stackrel{\mathcal{P}}{+}$ tracheostomy tube in the trachea, inflammatory 0 histological changes are noted in 24 to 48 hours (Atkins, 1964 : Cooper and Grillo, 1969). This $\stackrel{\Phi}{\Omega}$ inflammatory condition leads to a superficial $\mathbb{\otimes}$ tracheitis and mucosal ulceration within the first 0 week (Glas, King, and Lui, 1962). Continued 


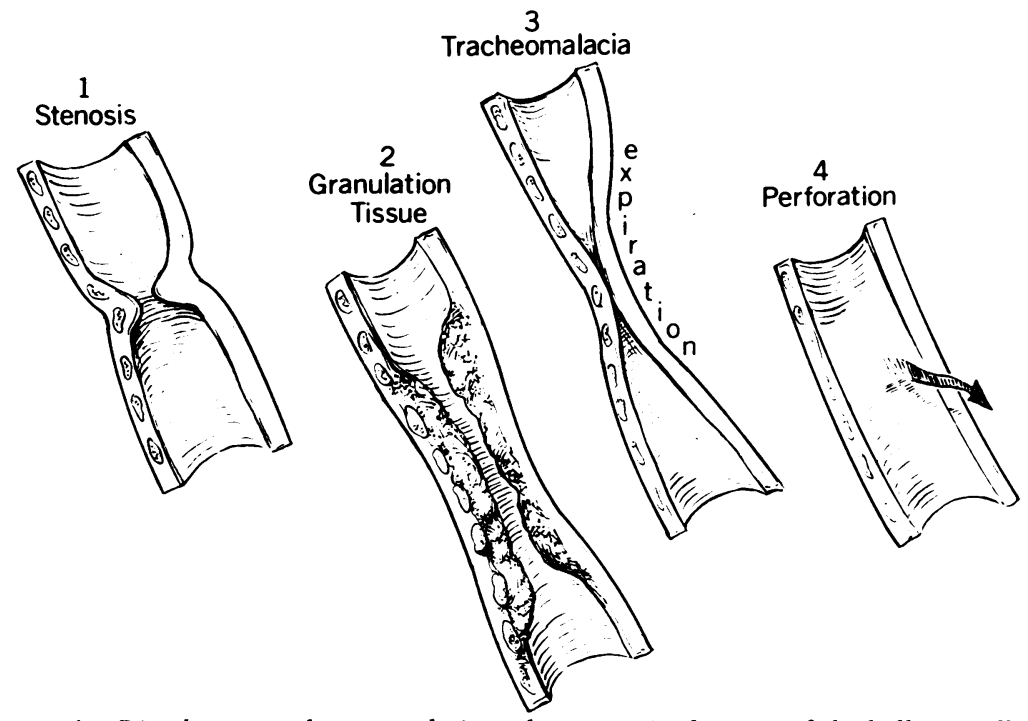

FIG. 1. Distal post-tracheostomy lesions that occur in the area of the balloon cuff.

inflammation and superimposed infection may result in deeper mucosal ulcerations and exposure of the underlying cartilage in one to three weeks (Pearson et al., 1968). As the cartilage is exposed chondritis is seen. In two to three weeks this may cause fragmentation and total loss of the cartilagenous supporting structures. The end results are a combination of destructive and reparative processes coexisting in a segment of the trachea (James et al., 1970a).

The proximal tracheal lesion probably begins from the mechanical abrasion of the tracheal mucosa by the tracheostomy tube (McLellano, 1965). As the tube is inserted the outer convexity rests upon the anterior tracheal wall superiorly and the posterior tracheal wall inferiorly (Fig. $2 \mathrm{a}, \mathrm{b})$. When the tracheostomy tube is then attached to a mechanical breathing apparatus, the rigidity of this placement is enhanced as are the shearing forces between the tracheostomy tube and the tracheal mucosa (Lu, Tamura, and Koobs, 1961 ; Meade, 1961). Drying of the tracheal mucosa (because the upper respiratory passages that provide moisture are bypassed) and superimposed infection are undoubtedly contributory (Stiles, 1965). Attempts at healing and repair in the area of the tracheostomy site may be seen as masses of graulation tissue formation, especially on the anterior wall, and asymmetrical or cicatricial stenosis due to fibrous healing (Fraser and Bell, 1967).

The distal post-tracheostomy lesions most often occur 1.5 to $3.0 \mathrm{~cm}$ distal to the site of tracheostomy insertion and usually involve a 2.5 to 3.0 $\mathrm{cm}$ segment of the trachea (Deverall, 1967 ; Flavell, 1959; Grillo, 1968). The character of these lesions is related to the type of ballon cuff used. The rigidity of the balloon wall determines the amount of intraluminal pressure necessary to ensure an airtight system required for assisted respiration (Grillo, 1969 ; Lu et al., 1961). To prevent air leakage around the balloon cuff, with a thick-walled balloon intraluminal pressures of 180 to $250 \mathrm{mmHg}$ must be maintained. Only 10 to $30 \mathrm{mmHg}$ pressure is required if a thin-walled balloon is used (Cooper and Grillo, 1969 ; James et al., 1970b). The mechanism by which thick- and thin-walled cuffs attain an airtight seal are also different. A thick-walled balloon distorts the trachea to conform to the shape of the balloon. Thin-walled balloons will easily adapt or conform to the irregularly shaped space between the tracheal lumen and the tracheostomy tube (Gibson, 1967). The lateral pressure of the balloon cuff acting as a right-angle force to the mucosa of the tracheal wall is the major cause of distal posttracheostomy lesions (Grillo, 1968 ; Johnston, Wright, and Hercus, 1967). The lateral force of the balloon cuff exceeds capillary pressure in the tracheal mucosa and causes necrosis and superficial mucosal loss. Disruption of the mucosal barrier facilitates superimposed infection, chondritis, and loss of supporting structures. With loss of the more rigid cartilagenous support, further 


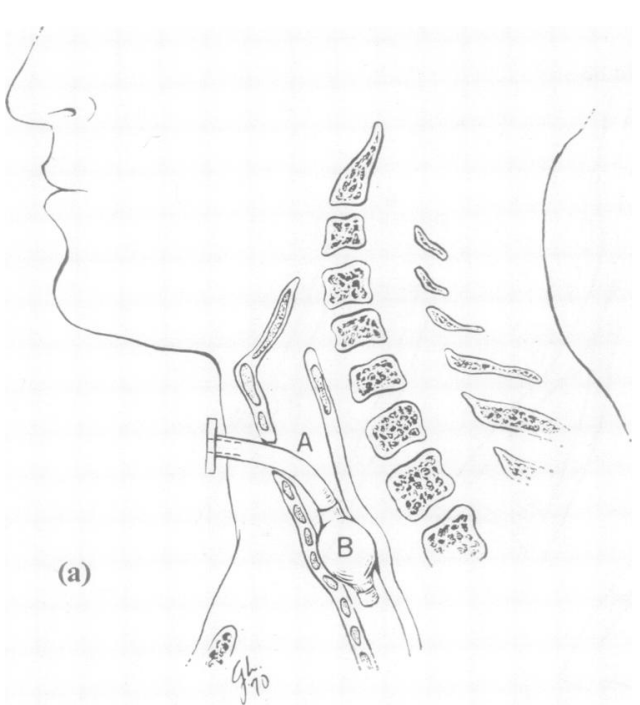

FIG. 2 (a). Anatomical relationship of trachea to cuffed tracheostomy tube. (A) Area of proximal lesion; anteriorly and superiorly tracheostomy tube rests against anterior tracheal wall. Posteriorly and inferiorly convexity of tracheostomy tube presses against posterior tracheal mucosa. (B) Area of distal lesions at site of balloon cuff. (b). Oblique radiograph demonstrating area of proximal lesions. Vertical arrow indicates area of granuloma formation just superior to tracheostomy tube. Large arrow at convexity of tracheostomy tube as it lies adjacent to posterior tracheal wall. Row of small horizontal arrows demonstrates distal anterior tracheal wall.

overflation of the balloon cuff is necessary to produce an airtight seal.

Perforation of the tracheal wall is most often a result of localized pressure necrosis of the distal tracheostomy tube tip (Flege, 1967). The balloon cuff may be placed too proximally, causing the balloon to act as a fulcrum. The distal tracheostomy tube tip presses into the tracheal wall causing localized necrosis and perforation. Perforation may result in a tracheo-oesophageal fistula or, more acutely, mediastinitis.

CLINICAL SYMPTOMS The onset of severity of clinical symptoms is highly variable and depends upon the type of lesion present, as well as on the patient's awareness and physical activity. Patients are usually removed gradually from the respirator, then the balloon-cuffed tracheostomy tube is replaced by one without a balloon cuff (sometimes followed by a fenestrated type). Later, the tube is removed and the tracheostomy site is eventually closed (James et al., 1970). However, this sequence of events may be dramatically altered if posttracheostomy lesions are present.

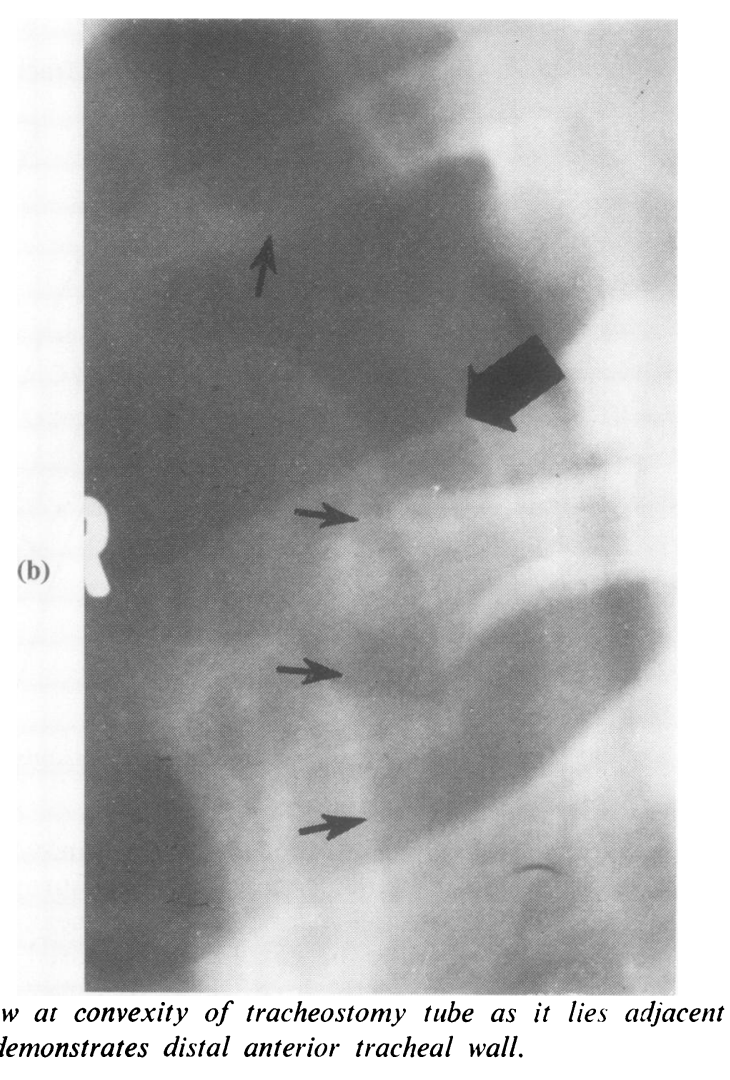

Granulation tissue formation at the tracheostomy site may effect a ball-valve type of mechanism causing intermittent obstructive episodes of acute respiratory distress (James et al., 1970a, b). After removal of the tracheostomy tube, fibrous healing may occur over a period of months, leading to narrowing of the tracheal lumen due to stenosis. This narrowing, in our experience, may occur distally or proximally but is most often encountered at the site of the balloon cuff. The patient may have a variable symptom-free period after removal of the tracheostomy tube and later experience difficulty in raising secretions (Grillo, 1968 ; Grillo, 1969). As the granulation tissue formation and healing by fibrosis progresses, the patient may experience respiratory stridor (Pearce and Walsh, 1961).

In patients with significant tracheomalacia, at the time of removal of the cuffed tracheostomy tube, immediate obstructive symptoms may be observed (Johnston et al., 1967). The loss of rigidity of the tracheal wall may result in paradoxical motion and apposition of the tracheal walls with respiration. Often the walls are held 
open by the balloon cuff. Removal will result in collapse of the tracheal walls and closure of the tracheal lumen. Immediate reinsertion of the tracheostomy tube to restore the airway is necessary.

Perforation of the tracheal wall can cause immediate acute symptoms. Concomitant erosion of a large thoracic vessel or mediastinitis are lifethreatening circumstances that require immediate therapy and do not allow a lengthy pretreatment evaluation.

RADIOLOGICAL EVALUATION The starting point in any radiological evaluation of the tracheobronchial tree is a properly exposed upright postero-anterior and lateral chest radiograph. A survey examination of this type may not optimally demonstrate the tracheal lesion, but will decrease the probability of overlooking a lesion in other portions of the respiratory tract. The

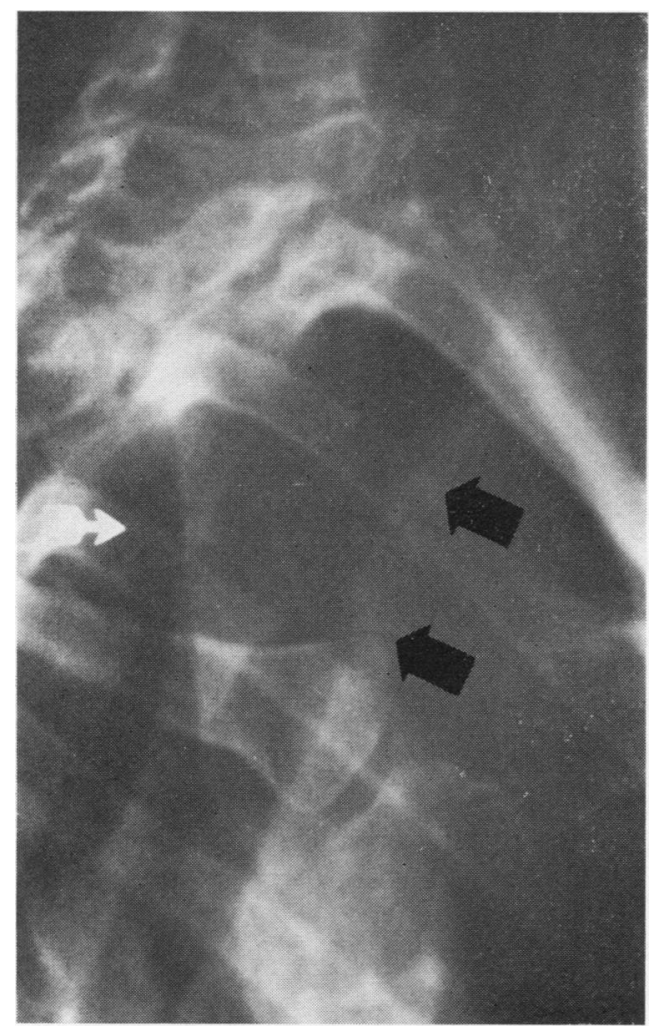

FIG. 3. Oblique radiograph showing area of tracheomalacia (large double arrows), inward deviation of tracheal walls, and narrowing of tracheal lumen with respiration. White arrow indicates patient's right side.

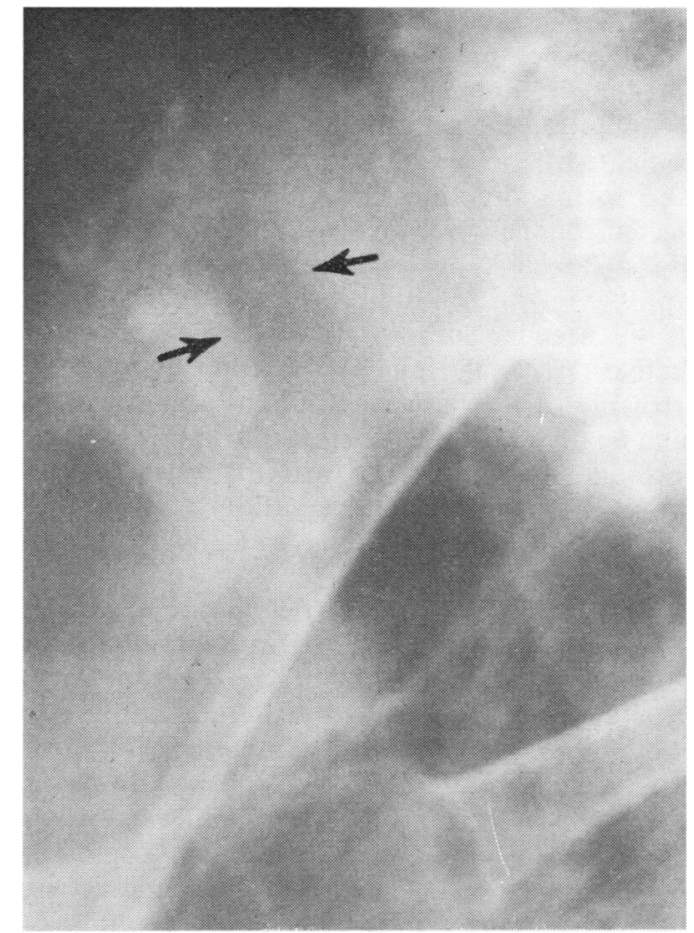

FIG. 4. Oblique radiograph of area of granulation tissue formation. Anterior tracheal wall is irregular and encroaches upon tracheal air column. Minimal inward deviation of the posterior tracheal wall.

chest radiograph should be followed by fluoroscopic examination of the trachea and bronchi as well as evaluation of diaphragmatic and chest wall motion. At fluoroscopy selected radiographs of the particular area of interest can be obtained. Selected fluoroscopic radiographs during inspiration and expiration are useful in determining the effective tracheal lumen in the area of stenosis or granulation tissue formation. Radiographs during respiration under fluoroscopic control are most useful in the assessment of patients with suspected tracheomalacia (James et al., 1970). The loss of cartilagenous support causes that tracheal segment to collapse with inspiratory effort (Fig. 3). Paradoxical motion of the tracheal wall can be observed. If the balloon cuff is in place at the beginning of the evaluation, the patient should be fluoroscoped to compare the diameter of the balloon to the width of the tracheal air column. This is done to determine if the balloon is overinflated and greatly exceeds the diameter of the tracheal lumen and if the balloon is holding the 
trachea open. The balloon cuff is then removed but only after preparations have been made for immediate reinsertion.

Normally the tracheal walls will appear as thin, parallel, soft tissue densities. With granulation tissue formation, thick irregular masses may be detected fluoroscopically and their extent carefully delineated by appropriate selected radiographs (Fig. 4). With fibrosis the tracheal walls will be seen to deviate inward, narrowing the effective tracheal lumen. At fluoroscopy this narrowing of the lumen will not appear to change dramatically with respiration. If tracheomalacia is present, the air column within the trachea will change markedly with respiration and the soft tissue of the tracheal walls will move outward and inward.

Following fluoroscopic evaluation of the tracheobronchial tree selected radiographs of the abnormal areas should be obtained. For proximal lesions at the tracheostomy site a lateral neck radiograph using soft tissue technique is often the most useful projection (Fig. 5). Lesions involving the lateral wall are often depicted only on the anteroposterior radiograph, however. With the typical granulation mass formation, often referred to as a 'granuloma ball', the amount of air space in the trachea posterior to this lesion should be

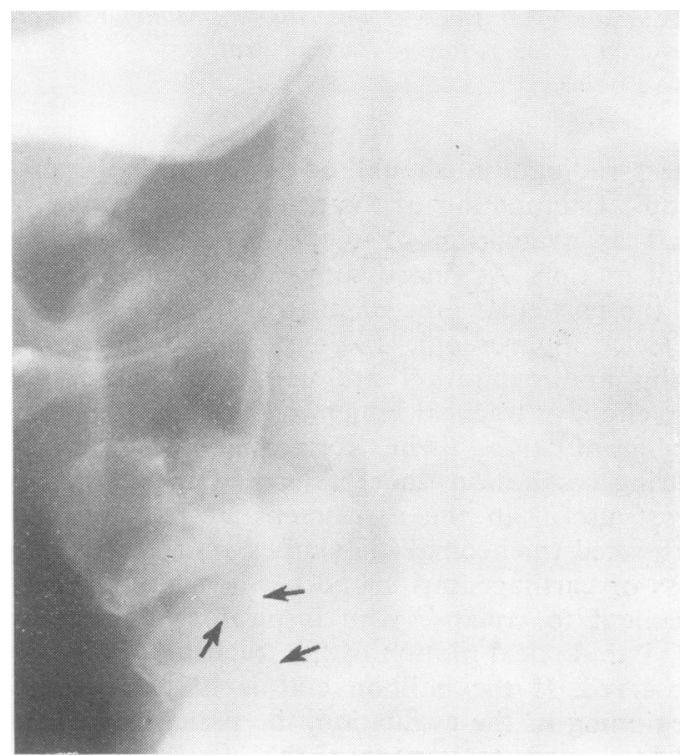

FIG. 5. Lateral neck radiograph: soft tissue detail optimizes demonstration of the granuloma mass (oblique arrow') and slight inward deviation of the posterior tracheal wall (horizontal arrows).

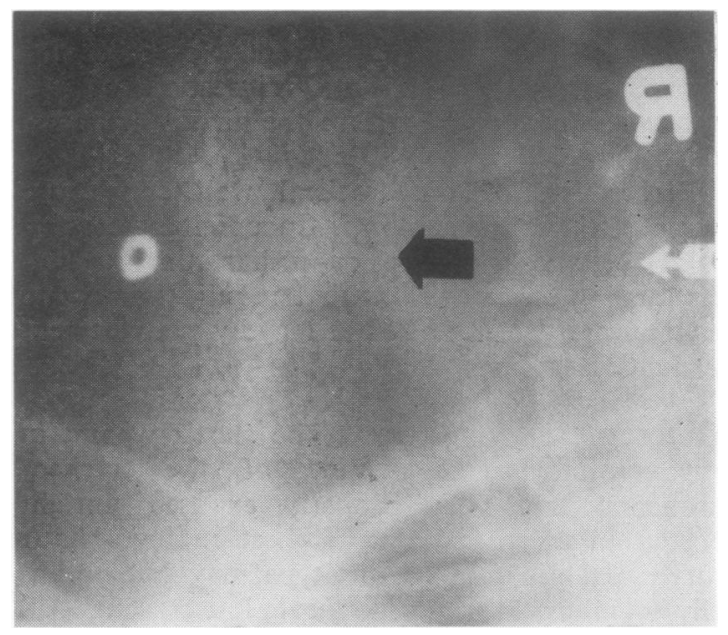

FIG. 6. Oblique radiograph demonstrates relation of granuloma mass to posterior tracheal wall. Small lumen is indicated (arrow).

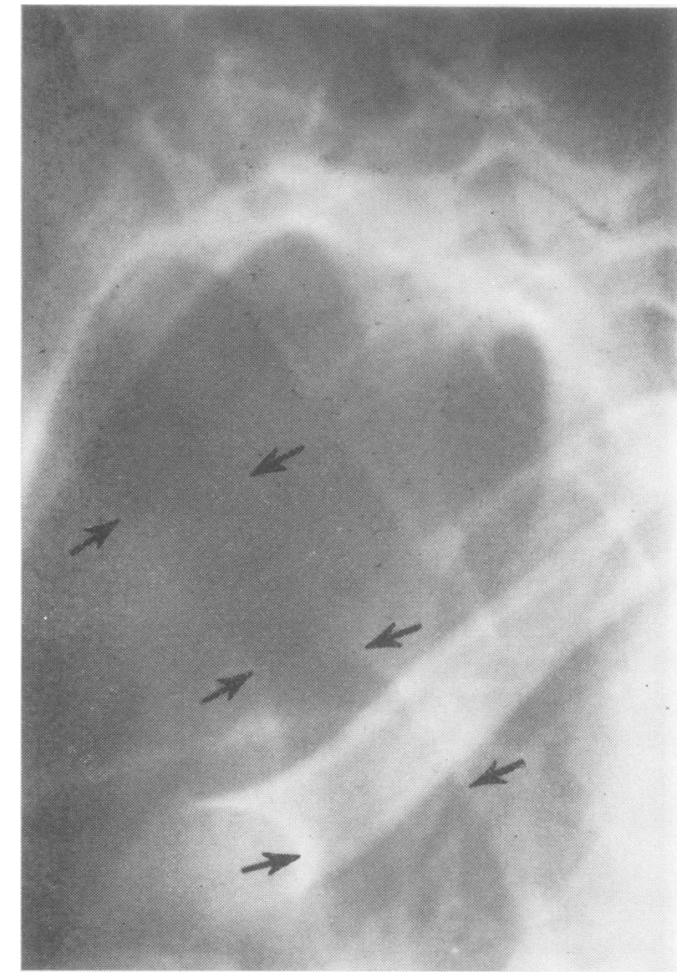

FIG. 7. Oblique radiograph eliminates overlying osseous structures and optimally demonstrates minimal inward deviation of tracheal walls. 
determined (Fig. 6). Occasionally, oblique views must be obtained to radiograph the 'granuloma ball' and posterior tracheal air column tangentially. Also the presence of associated stenosis or tracheomalacia must be recognized to decide whether or not transtracheal removal of the granulation mass will provide definitive therapy (Pearson et al., 1968).

Distal tracheal lesions in the area of the balloon cuff are often obscured by overlying osseous and soft tissue structures. Oblique radiographs provide optimum delineation of the lesions (Fig. 7) which may not be apparent with postero-anterior and lateral radiographs even using a high kilovoltage technique.

Laminography is a very useful radiographic modality to enhance anatomical detail at a selected depth and location. Multiple radiographs at depths of optimal resolution in more than one plane often provide an accurate assessment of the size, configuration, and location of a lesion (Fig. 8). Laminograms are useful in depicting the 'mass-like' nature of granulation tissue formation (James et al., 1970). Occasionally, dystrophic calcification may be seen by laminography when it is not seen on plain radiographs.

We have been very conservative in our attitude towards the use of contrast media in the radiographic evaluation of tracheal lesions (James et al., 1970). Positive contrast compounds that con- tain iodine or barium may, at times, cause deleterious effects on the respiratory function of patients with post-tracheostomy lesions. Large

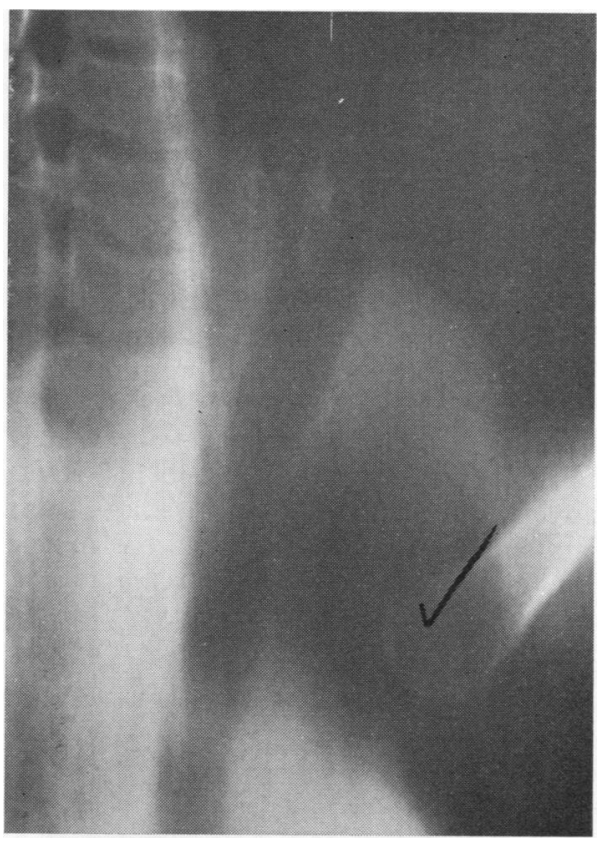

FIG. 8. Laminogram optimizes visualization of the tracheal lumen in the plane of focus.

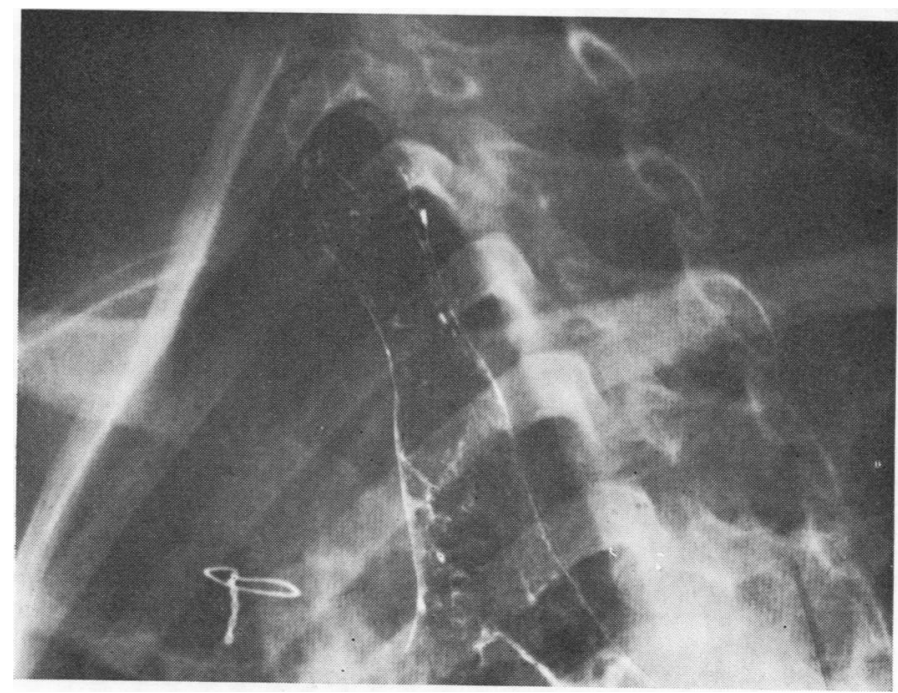

FIG. 9. Oblique fluoroscopic radiograph with tantalum obtained during inspiration. There was increased mobility of the posterior tracheal wall indicating tracheomalacia (after open heart surgery). 
amounts of radiographic contrast will severely compromise the airway in these patients with disastrous results. In addition, oily contrast agents coat the mucosa poorly and may induce acute inflammation locally. Therefore, we have in the past recommended their use only when other methods have failed and then in very small amounts. Because of these objections to iodine and barium contrast agents we, among others, have investigated the use of powdered metallic tantalum (Hinchcliffe et al., 1970).

Tantalum is chemically inert, non-toxic, and very radiodense. The finely powdered tantalum $(0.5 \mu$ particle size) coats the mucosa and adheres to its surface despite forceful respiratory movements (Nadel, Wolfe, and Graf, 1968). The lack of toxicity as well as the small volume necessary for a tracheogram $(0.2$ to $0.5 \mathrm{ml})$ has resulted in no adverse effect in any patient we have examined.

Tantalum is cleared rapidly via mucociliary action (Nadel et al., 1970). Delayed radiographs taken six hours after the tracheogram have shown no residual tantalum. After proper premedication

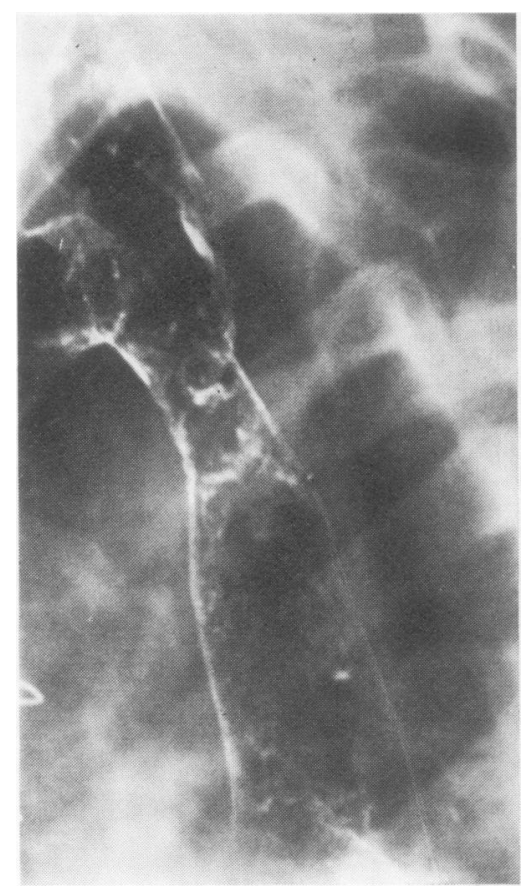

FIG. 10. Oblique radiograph demonstrates a sharply defined area of tracheal stenosis. The granular appearance of the tantalum in the area of stenosis represents marked irregularity of the mucosa with leucoplakia (after open heart surgery). and local anaesthesia, tantalum is insufflated into the trachea through a catheter. The deposition of tantalum is controlled fluoroscopically. Fluoroscopic and conventional radiographs including oblique, frontal, and lateral views are obtained. Fluoroscopic radiographs during respiratory movements have shown increased mobility of segments with tracheomalacia (Fig. 9). This has also been documented by ciné examination. The delineation of the structural abnormality as well as the fine mucosal detail have been excellent (Fig. $10)$.

\section{CONCLUSION}

Decisions regarding the proper therapy for posttracheostomy lesions depend upon proper assessment of the alterations of anatomy and physiology. Pulmonary function studies and bronchoscopy are mandatory and provide valuable information. Many functional and structural determinations may be made by proper use of radiological techniques. Static radiographs, laminograms, and contrast tracheograms with fluoroscopic monitoring allow anatomical and physiological assessment using the same diagnostic technique. A three-dimensional delineation of the abnormalities is possible, thus allowing for proper planning of the surgical approach. Communication between the physician primarily responsible for the care of the patient and his radiological consultant will add greatly to the accuracy of this evaluation.

\section{REFERENCES}

Atkins, J. P. (1964). Tracheal reactions following the use of cuffed tracheotomy tubes. Ann. Otol. (St. Louis), 73, 1124.

Cooper, J. D., and Grillo, H. C. (1969). The evolution of tracheal injury due to ventilatory assistance through cuffed tubes: a pathologic study. Ann. Surg., 169, 334.

Deverall, P. B. (1967). Tracheal stricture following tracheostomy. Thorax, 22, 572.

Flavell, G. (1959). Resection of tracheal stricture following tracheotomy, with primary anastomosis. Proc. roy. Soc. Med., 52, 143.

Flege, J. B., Jr. (1967). Tracheoesophageal fistula caused by cuffed tracheostomy tube. Ann. Surg., 166, 153.

Fraser, K., and Bell, P. R. F. (1967). Distal tracheal stenosis following tracheostomy. Brit. J. Surg., 54, 302.

Gibson, P. (1967). Aetiology and repair of tracheal stenosis following tracheostomy and intermittent positive pressure respiration. Thorax, 22, 1.

Glas, W. W., King, O. J., Jr., and Lui, A. (1962). Complications of tracheostomy. Arch. Surg., 85, 56. 
Grillo, H. C. (1968). The management of tracheal stenosis following assisted respiration. J. thorac. cardiovasc. Surg., 57, 52.

(1969). Surgical approaches to the trachea. Surg. Gynec. Obstet., 129, 347.

Hinchcliffe, W. A., Zamel, N., Fishman, N. H., Dedo, H. H., Greenspan, R. H., and Nadel, J. (1970). Roentgenographic study of the human trachea with powdered tantalum. Radiology, 97, 327.

James, A. E., Jr., Macmillan, A. S., Eaton, S. B., Grillo, H. C. (1970a). Roentgenology of tracheal stenosis resulting from cuffed tracheostomy tubes. Amer. J. Roentgenol., 109, 455.

MacMillan, A. S., Jr., Eaton, S. B., Janower, M. L., and Grillo, H. C. (1970b). Radiological considerations of stenosis and granuloma at the site of tracheostomy. Radiology, 96, 513.

Johnston, J. B., Wright, J. S., and Hercus, V. (1967). Tracheal stenosis following tracheostomy. $J$. thorac. cardiovasc. Surg., 53, 206.

Lu, A. T., Tamura, Y., and Koobs, D. H. (1961). The pathology of laryngotracheal complications. Arch. Otolaryng., 74, 323.
McLellano, R. M. (1965). Complications of tracheostomy. Brit. med. J., 2, 567.

Meade, J. W. (1961). Tracheotomy-its complications and their management. A study of 212 cases. New Engl. J. Med., 265, 519.

Murphy, D. A., MacLean, L. D., and Dobell, A. R. C. (1966). Tracheal stenosis as a complication of tracheostomy. Ann. thoracic Surg., 2, 44.

Nadel, J. A., Wolfe, W. G., Graf, P. D. (1968). Powdered tantalum as a medium for bronchography in canine and human lungs. Invest. Radiol., 3, 229.

Youker, J. E., Zamel, N., Austin, J. H. M., Hinchcliffe, W. A., Greenspan, R. H. and Wright, R. R. (1970). Powdered tantalum: a new contrast medium for roentgenographic examination of human airways. $\mathrm{New}^{-}$ Engl. J. Med., 283, 281.

Pearce, D. J., and Walsh, R. S. (1961). Respiratory obstruction due to tracheal granuloma after tracheostomy. Lancet, 2, 135.

Pearson, F. G., Goldberg, M., and da Silva, A. J. (1968). Tracheal stenosis complicating tracheostomy with cuffed tubes. Arch. Surg., 97, 380.

Stiles, P. J. (1965). Tracheal lesions after tracheostomy. Thorax, 20, 517. 